\title{
La sensibilidad del profesorado hacia el modelo de educación intercultural: Necesidades, situación actual y propuesta de un instrumento de medida
}

Teachers' sensitivity to the model of intercultural education: Needs, current situation and proposal of a measuring instrument

\section{A sensibilidade dos professores ao modelo de educação intercultural: Necessidades, situação atual e proposta de um instrumento de medição}

\author{
Olga González, ${ }^{a}$ Llarela Berríos, $^{b}$ María Rosa Buxarrais ${ }^{c}$
}

${ }^{\text {a}}$ Universitat de Barcelona, Departamento de Didáctica de la expresión musical y corporal. Passeig. de la Vall d'Hebron, 171 AP: 08035 Barcelona, España. Telf.: (34)934035134. Correo electrónico: ogonzalez@ub.edu

bUniversidad de los Andes, Instituto de Ciencias de la Familia. Av. San Carlos de Apoquindo 2200, Las Condes, Santiago de Chile. Telf.: (562) Correo electrónico: 1larela.berrios@uandes.cl

'Universitat de Barcelona, Departamento de Teoría e Historia de la educación. Passeig. de la Vall d'Hebron, 171 AP: 08035 Barcelona, España. Telf.: (34)934035225. Correo electrónico mrbuxarrais@ub.edu

\begin{abstract}
RESUMEN
Durante los últimos años, en Chile se está produciendo un fenómeno de inmigración proveniente de otros países que está conformando un nuevo contexto social multicultural. Por supuesto, las aulas reflejan esta pluralidad de culturas, a las que el sistema educativo debe dar respuesta, principalmente, a través de la formación del profesorado. El artículo se estructura en dos partes: en un primer momento, y aprovechando la experiencia reciente de países como España en esta temática, se presentarán diferentes actitudes de la comunidad educativa ante la educación intercultural; en una segunda parte se ofrecen pautas de cómo debería ser la formación ideal del profesorado en este ámbito y, finalmente, se propone una herramienta de recogida de información que sirve para medir la sensibilidad del profesorado y la posibilidad de plantear una formación permanente de los docentes, una sólida competencia intercultural, así como la detección de grupos de trabajo para liderar acciones transformadoras en las escuelas.
\end{abstract}

Palabras clave: formación del profesorado, educación intercultural, cuestionario.

\begin{abstract}
In recent years, Chile is producing a phenomenon of immigration from other countries that are shaping a new multicultural social context. Of course, the classrooms reflect this plural-ity of cultures, so that the education system must answer primarily through teacher training. This article is structured in two parts: at first, taking advantage of the recent experience of countries like Spain in the area, it presents different attitudes of the educational community to intercultural education, in a second part it provides guidelines for how it should be ideal training for teachers in this area and, finally, we propose a data collection tool used to measure the sensitivity of teachers and raise the possibility of continuing education of teachers, a strong intercultural competence as well as the detection of working groups to lead transformative actions in schools.
\end{abstract}

Key words: teacher training, intercultural education, questionnaire.

\section{RESUMO}

Nos últimos anos, o Chile está produzindo um fenômeno da imigração de outros países que estão moldando um contexto multicultural novo social. Claro, as salas de aula refletem essa pluralidade de culturas, de modo que o sistema educativo deve responder principalmente através da formação de professores. $\mathrm{O}$ artigo está estruturado em duas partes: num primeiro momento, aproveitando a experiência recente de países como a Espanha na área, 
apresentará diferentes atitudes da comunidade educacional para a educação intercultural, numa segunda parte fornece orientações de como deve ser treinamento ideal para professores nesta área e, finalmente, propor um instrumento de coleta de dados utilizado para medir a sensibilidade dos professores e aumentar a possibilidade de formação continuada de professores, uma forte competência intercultural, bem como a detecção de grupos de trabalho para levar ações transformadoras nas escolas.

Palavras chave: formação de professores, educação intercultural, questionário.

\section{INTRODUCCIÓN}

No cabe duda de que la educación en contextos multiculturales es hoy día una temática nuclear dentro de los estudios pedagógicos, como demuestran los diversos artículos que recogen distintas revisiones, investigaciones y propuestas en este ámbito, entre los que destacamos a Rubio (2009), Ferräo (2010) y Aranda (2011). En los últimos años, en América Latina en general y, en particular en Chile, por su especial situación de estabilidad política y económica, la inmigración ha aumentado de manera espectacular (un $50 \%$ desde 2004 a 2010), situándose actualmente en un 2,5\% del total de la población, con un $68 \%$ de los inmigrantes provenientes de Sudamérica. Dicha situación probablemente vaya en aumento, por eso se hace necesario una propuesta de medidas educativas que den respuesta a estos nuevos contextos multiculturales.

El modelo de educación intercultural que pone el énfasis en el concepto de cultura como un proceso dinámico y de "suma", es, sin duda, el ideal educativo al cual aspirar. Nuestro concepto de educación intercultural incluye también la relación entre culturas dentro del mismo territorio. Así, la educación intracultural sería, obviamente, una característica más dentro del concepto más amplio de interculturalidad, de acuerdo con las orientaciones del Ministerio de Educación Chileno, que Rubio (2009) nos detalla.

Definitivamente, se trataría de superar los modelos educativos homogenizadores, de carácter asimilacionista y compensatorio que igualaban "diferencia" a "deficiencia" (Ferräo, 2010), que coinciden también con lo acontecido en otros países como España, donde se ha dado un proceso parecido en los últimos años (CIDE, 2005).

La modificación del enfoque pedagógico tiene como objetivo evitar situaciones de conflicto en la escuela, donde el niño-joven de hoy, que será el adulto de mañana, no debería ser abocado a la disyuntiva de optar por una situación de asimilación o exclusión, sino por una integración inclusiva donde todas las personas tengan opción de ser reconocidas y aceptadas y con la libertad de desarrollar su propia identidad cultural.

La adopción del modelo educativo intercultural se basa, necesariamente, no sólo en la disposición de nuevas leyes transformadoras -incluida la revisión de los currículums oficiales- sino, y principalmente, en el convencimiento del profesorado -principal agente educativo- de la necesidad de este cambio. Nos encontramos pues con la necesidad de que el profesorado reciba una formación específica que le permita acometer las transformaciones previstas y superar sus propias actitudes y prejuicios previos. La formación inicial del profesorado será entonces un pilar imprescindible en este proceso (Rubio, 2009; Aranda, 2011 y Jordán, 2004).

Además de la formación inicial del profesorado, no debemos olvidar la necesidad de una formación permanente en el mismo sentido, ya que no únicamente es necesario que los nuevos pedagogos se formen en este nuevo modelo, sino que el profesorado en activo modifique su práctica educativa de forma coherente. Es con esta última intención que presentamos una revisión de actitudes del profesorado en relación a dicha 
temática. La muestra corresponde a maestros y maestras españolas entre 1999 y 2005 , justo cuando se planteaba en este país la necesidad de la formación del profesorado en el nuevo modelo educativo, y que nos permiten tomar conciencia de la situación inicial en cuanto al colectivo. Detallaremos, además, las pautas de formación pedagógica desde este enfoque, para ser adoptadas tanto en la formación inicial como en la formación permanente del profesorado.

Finalmente, proponemos un instrumento de recogida de información (cuestionario), adaptado de González Mediel (2008), cuya finalidad es medir la sensibilidad intercultural del profesorado en activo. Los objetivos que se pretendían en la investigación original fueron: 1) conocer a fondo el pensamiento del profesorado de un centro educativo con el cual se quería iniciar una transformación curricular para, 2) adoptar el modelo intercultural en la escuela. Los resultados permitieron crear un grupo de trabajo que liderara el cambio educativo en el centro.

\section{LA COMUNIDAD EDUCATIVA Y LA EDUCACIÓN INTERCULTURAL}

Antes de conocer las directrices que deberían lograrse por parte del profesorado para conseguir llevar a cabo un cambio educativo desde la perspectiva intercultural, es necesario que conozcamos qué actitudes previas con respecto a la diversidad cultural tiene la comunidad educativa.

\subsection{ACTITUDES DE LA COMUNIDAD EDUCATIVA ANTE LA DIVERSIDAD CULTURAL}

Una investigación del Colectivo IOE (1999) nos permite saber que el profesorado adopta cinco tipos de actitudes ante la diversidad cultural y que no todas son proclives a un modelo intercultural.

1) Una primera actitud sería la de rechazo hacia los diferentes, donde la diversidad se percibe como un peligro del que conviene huir, en la línea de poner bajo sospecha al diferente, al extranjero, al contrario de aquello que es conocido. Así pues, se ponen trabas y dificultades para su ingreso en el centro, reduciendo al máximo el contacto con ellos. Esta actitud se da tanto entre los responsables de los centros como entre los padres y madres del alumnado, que aconsejan a hijas e hijos el no acercarse a los "diferentes", llegando incluso a cambiarlos de escuela, si su presencia se constata demasiado numerosa.

2) La segunda opción que aparece es la de ignorar a los diferentes. Es desde esta concepción donde se justifica que el alumnado sea un grupo homogéneo y se proponga un modelo único de currículum. Todo el mundo tiene que adaptarse a las pautas establecidas para todos. Es una perspectiva que se justifica desde la no discriminación, puesto que considera a todo el alumnado de una misma manera.

3) Una tercera variante es la que contempla un reconocimiento de la necesidad de educar de una forma diferente al alumnado recién llegado, pero sin cambiar nada del currículum común, sino abriendo espacios para una atención particularizada, en la línea de la educación compensatoria. Esta es la actitud que parece contar con más apoyo por parte de los y las maestras, sobre todo a la enseñanza infantil y primaria (Bartolomé, 1997b).

4) La cuarta opción sería la más adecuada para un enfoque educativo intercultural, puesto que parte de la adaptación del currículum en función del conjunto del alumnado e incorpora elementos de diferentes perspectivas socioculturales. Este enfoque es todavía 
muy reciente en las escuelas españolas por diferentes razones, tanto por la escasez de materiales didácticos y experiencias, la falta de formación específica del profesorado o bien por el peso específico de la tradición escolar homogeneizadora hasta tiempos bastante recientes.

5) La última opción, identificada en países con más tradición en este ámbito (Banks, 2004), es la considerada como educación antirracista, similar a la educación intercultural, pero con un alcance más amplio de tipo social, puesto que considera que sólo en la escuela no se puede solucionar el problema de la exclusión.

Jordán (1996), por otro lado, especifica que, en el contexto español, más de la mitad del profesorado encuestado presentaba una predisposición abiertamente negativa o indiferente en relación a la promoción y defensa de las minorías étnicas y sus respectivas identidades culturales (actitudes 1 y 2). Estos resultados son plenamente coincidentes con los que fueron obtenidos por Bartolomé (1997b). Jordán (1996) nos muestra su escepticismo respecto a los datos obtenidos, y más bien se inclina a pensar que todavía es más elevado el número de profesorado predispuesto negativamente, además de recordarnos que estas creencias y actitudes reales son las que más influyen en su práctica cotidiana. Parece que entre los y las profesionales de la educación hay todavía un sentimiento generalizado de que la diversidad cultural es una realidad compleja para la que no se está preparado. Conocemos con el Colectivo AMANI (2005) varias conclusiones que reflejan este sentir general:

- La diversidad en las aulas se vive como un aspecto ajeno que viene a complicar la realidad educativa, el profesorado se enfrenta como puede y sólo si existe una motivación personal hay una preocupación para formarse o buscar alternativas.

- Aunque hay una conciencia de que la postura ante la diversidad cultural no tiene que ser de tipo asimilatorio, se sigue identificando el "conflicto" con el alumnado inmigrante o de grupos étnico-culturales diferentes, por lo cual las acciones se dirigen únicamente a estos colectivos.

- La comunidad educativa no cree estar preparada para atender las diferentes realidades que comportan otras culturas y el resultado ante las pocas alternativas es la indiferencia. Son realidades todavía no asumidas, ante las cuales no hay opciones y el profesorado encuentra un vacío.

- La flexibilidad del currículum se entiende como una ventaja a la hora de afrontar la diversidad, aún así, las acciones que se introducen acostumbran a ser puntuales, todavía no se trabaja transversalmente. Jornadas, discusiones y actividades concretas y aisladas se imponen al hecho de incluir la interculturalidad en el día a día.

- Las soluciones se tienen que buscar a la fuerza por el camino de la transformación de estructuras mentales y físicas. Hay cierta resistencia a cambiar esquemas educativos, pero lo cierto es que el modelo actual no funciona ante la realidad multicultural, así que se debe ir más allá y probar nuevas fórmulas: situarse en la piel del otro y, desde aquel punto, hacer nuevos planteamientos.

Otra investigación coordinada por Aguado en 2004 (CIDE 2005), "Proyecto Inter. Formación de profesores en educación intercultural. Análisis de necesidades", vuelve a poner de relieve que la formación del profesorado es optativa y puntual. Se continúa planteando desde enfoques compensatorios y se centra más en contenidos que en actitudes y competencias. Es necesario pues integrar la formación en educación intercultural en los planes de estudio de los maestros y proporcionar recursos para el profesorado en ejercicio. 


\section{LA FORMACIÓN DEL PROFESORADO EN EDUCACIÓN INTERCULTURAL}

Es evidente que el profesor es el eje de la educación intercultural, incluso en mayor medida que en otros aspectos educativos, donde los materiales de apoyo a la docencia reflejan los objetivos que se demandan muy claramente, cosa que no ocurre con la E.I. Así que, efectivamente, el profesorado se convierte en el motor del cambio educativo. El profesorado encuentra sentido al cambio cuando se fundamenta en su propio conocimiento práctico y en su experiencia personal (Bartolomé, 1997a). Por eso hace falta un nuevo modelo de formación del profesorado que lo prepare para desarrollar su actuación profesional en contextos caracterizados por la diversidad cultural.

Aunque la necesidad es clara (Jordán, 2004), los datos reflejan la falta de formación específica del profesorado en este ámbito, ya que se había detectado previamente en los y las docentes esta necesidad junto a una cierta ansiedad al respeto (Bartolomé, 1997a). El mismo Jordán (2004) nos recuerda que la educación específica que estos maestros han recibido no ha sido muy provechosa, en la medida que han sido conocimientos bastantes teóricos y poco útiles para su práctica cotidiana. Este mismo comentario se puede aplicar a los numerosos cursos de formación permanente que hoy en día se ofrecen en diversas instituciones.

Las facultades de formación del profesorado tienen un papel fundamental en la formación inicial de los y las maestras en cuanto a desarrollo de conocimientos, competencias, actitudes y didáctica (Sabariego, 2003; Rubio, 2009; y Aranda, 2011). Los y las formadoras de formadores deben promover innovaciones curriculares e incorporar contenidos de temas culturales orientados a la diversidad, pero también deben desarrollar nuevas estrategias de enseñanza-aprendizaje que induzcan al alumnado a la reflexión y al análisis intercultural. Así pues, este profesorado es el primero que tiene que asumir e integrar las creencias filosóficas, educativas y sociales que comporta un planteamiento intercultural. Pero este profesorado es también fruto de una educación monocultural, lo cual dificulta su capacitación para impartir una educación pluralista y formar a los y las futuras docentes en este sentido.

Desde otro enfoque, Jordán (2004) propone basar el modelo genérico de formación para el profesorado en tres vertientes: la cognitiva, la técnica y la actitudinal, de manera parecida a las que conocíamos por Torres (citado en Aranda, 2011).

a) Dimensión cognitiva. Tiene dos vertientes: la informativa y la crítica, ambas necesarias de cultivar separadamente. La dimensión cognitiva informativa estaría referida a unos conocimientos básicos sobre el entorno cultural del nuevo alumnado: socialización y educación previa, percepciones y expectativas de los progenitores sobre la educación escolar, los valores y pautas de conducta de las culturas de origen referidas al proceso académico, las dimensiones legales en el campo educativo y los rasgos más relevantes de las culturas más representativas del centro. Con este último punto se debe tener precaución con la selección de información, huir de conocimientos genéricos y buscar una visión realista y viva que ayude a superar prejuicios. En definitiva, sería recomendable, como dice Jordán (2004), contextualizar la información y no caer en la simplificación de considerar las identidades individuales como extensiones de una presunta identidad colectiva. La dimensión cognitiva crítica tiene que ser clarificadora. La mayoría de formación intercultural se basa en aspectos atomizados en forma de mosaico, no hay reflexión y debate sobre los temas. Por el contrario, se propone replantear las modalidades 
de formación para que incluyan la reflexión crítica sobre los contenidos: el relativismo cultural, la xenofobia, el concepto de identidad personal, la noción de diversidad. Si esta formación no es reflexiva y discutida, difícilmente se interiorizará por parte de los y las maestras, con lo cual la formación será superficial e ineficiente desde el punto de vista de la educación intercultural.

b) Dimensión técnico-pedagógica. El profesorado deberá adquirir competencias en este sentido que le permitan conseguir un repertorio de estrategias organizativas y didácticas que le faciliten la atención a la diversidad presente en los centros. Los aspectos más destacables podrían ser: 1) Elaborar currículums interculturales, desde la reflexión y el debate de todos los profesionales del centro educativo: el P.E.I. y, muy importante para favorecer un clima realmente intercultural, el currículum global del centro. 2) Adoptar estrategias que mejoren el éxito escolar de todo el alumnado, como por ejemplo la metodología cooperativa (Díaz-Aguado, 2003). Conocer los mecanismos organizativos y didácticos para acoger al alumnado que se incorpora a medio curso o sin escolarización previa en los centros. En este apartado destacan las aulas de acogida, pero que, como también tienen un cierto peligro de generar guetos, es recomendable debatir los pros y contras desde la reflexión crítica. Muy ligado al anterior punto, el profesorado aprenderá a diseñar y aplicar un plan de acogida para el alumnado minoritario de nueva incorporación. No se trata sólo del proceso burocrático, sino adoptar una actitud realmente "acogedora" que recoja como elementos importantes: la información cordial y clara a las familias sobre la escuela y el sistema educativo; diálogo constante con las familias sobre las expectativas y el desarrollo escolar de sus hijos e hijas; la facilitación de relaciones positivas entre todos los miembros de la comunidad escolar, aprender a afrontar y resolver conflictos de origen étnico-cultural, y ser capaz de analizar, modificar, seleccionar y crear materiales adecuados para atender la diversidad cultural del alumnado.

c) Dimensión actitudinal. Esta dimensión es quizás, la menos trabajada a nivel formativo y, como hemos visto, se percibe básica en la formación del profesorado en educación intercultural. Algunos consejos irían en la línea de: -Adoptar una actitud reflexiva. No hay nada escrito que tenga la solución para todos los casos posibles. Hay muchos esquemas que tienen que ser revisados, replanteados y modificados. Sería oportuno realizar un auto-conocimiento profesional que ayude a los y las maestras a tomar conciencia de sus propias convicciones, mediante el diálogo entre colegas, con guías didácticas específicas para cuestionarse la tarea educativa y con la anotación sistemática de incidentes críticos (Buxarrais, 1997). -Implicarse emocionalmente, puesto que no sirve una toma de conciencia fría en el plan cognitivo, las y los formadores tienen que conocer estrategias que se los permitan un cambio actitudinal profundo. En este sentido, se aconseja el enfoque socio-afectivo para que los haga vivir experiencias parecidas a las de su alumnado y el análisis de casos, preferentemente en grupo, que le permitan vivenciar creencias y actitudes de diferentes maestros y diferentes alumnos (Jordán, 2004). -Aprender desde la observación y la práctica conjunta con profesionales con competencias actitudinales y técnicas constatables. -Sustituir una visión problematizadora por otra abierta a la potencialidad enriquecedora de la diversidad; lograr la conciencia de la diversidad personal del alumnado, huyendo del mito de la homogeneidad; adoptar un talando reflexivo y críticoconstructivista respecto a las ideas y prácticas establecidas; replantear la tarea docente más educativa que instructiva; asumir una responsabilidad ético-profesional con todo el alumnado, huyendo de "derivar" a maestros especialistas el alumnado minoritario; estar 
dispuesto a trabajar conjuntamente y cooperativamente con todo el profesorado del centro; adoptar una actitud de constante formación y replanteamiento del modelo educativo intercultural y lograr verdaderas actitudes de tolerancia, valoración y reconocimiento hacia la diferencia. Por último, debemos destacar como principal consejo por el profesorado que quiere introducir este nuevo modelo educativo en su práctica el estar motivado/da. El profesorado tiene que estar ser motivada/do, adoptar actitudes y expectativas positivas, comprometerse en proyectos, implicar al máximo número de compañeros/as e intentar conocer lo mejor posible (cultural y personalmente) al alumnado nuevo (puntos fuertes y débiles). Este proceso es necesario para poder implicarse emocionalmente y conseguir mejores resultados, acostumbrarse a fomentar un aprendizaje significativo desde la práctica a la teoría, partiendo de semblanzas culturales y usando la autoevaluación como herramienta de estímulo para el alumnado.

Un proceso formativo eficiente para llegar a lograr un modelo que aglutinara los anteriores objetivos podría ser (Jordán, 2004):

a) Focalizar la preparación del profesorado en el propio centro educativo, con la finalidad de ajustar bien las necesidades sentidas con las reales y para implicar a todo el claustro en el cambio previsto.

b) Realizar la formación principalmente durante el tiempo lectivo, para evitar "añadir" programas y encaminarse más bien al cambio de funcionamiento del equipo docente.

c) Acercarse al modelo de investigación-acción, profesor como investigador del que hablan Carr y Kemmis (1988), en la propia práctica educativa. Esto permitiría trabajar de forma cooperativa a todo el profesorado siguiendo unos bucles de actuación y, a partir de un conocimiento teórico y de una reflexión inicial, plantear y desarrollar acciones que serían constantemente analizadas y que traerían a establecer nuevas acciones en un "continuum" de mejora educativa. El éxito de esta alternativa se apoyaría relacionando efectivamente la práctica con la teoría.

Este último punto acontece algo básico para conseguir encarar con éxito un cambio educativo. Díaz-Aguado (2003), Imbernón (1994) y Bartolomé (1997a) subscriben también la figura del docente como profesional reflexivo. Esto obliga a articular la propia formación con la actividad reflexiva e investigadora y la innovación en educación intercultural. El objetivo a lograr sería doble: la introducción de nuevas prácticas en el interior de la organización escolar y el cambio de actitudes del profesorado ante la educación intercultural (Colectivo AMANI, 2005).

\section{CUESTIONARIO PARA MEDIR LA SENSIBILIDAD INTERCULTURAL DEL PROFESORADO ${ }^{1}$}

El cuestionario se compone de una serie de ítems en torno a un determinado problema o cuestión sobre el cual se desea investigar. Las respuestas se darán por escrito. Dado que uno de los objetivos del trabajo de investigación de González Mediel era caracterizar al profesorado respecto a su sensibilidad hacia el modelo educativo intercultural, la metodología del estudio contemplaba este instrumento como técnica de recogida de datos de

Para su posible aplicación en diversos entornos, hemos modificado diversos aspectos formales en esta versión y entendemos que puede ser adaptado, posteriormente, según necesidades. 
carácter cuantitativo en la primera fase de la investigación. Los objetivos a lograr a través del cuestionario de caracterización son: 1. Conocer los datos precisos de cada uno de los/las maestros/as de la escuela tanto personales como profesionales, con el objetivo de evaluar efectivamente sus respuestas. 2. Averiguar la opinión del profesorado en cuanto al modelo educativo de la escuela: ¿cuál tiene? y ¿cuál debería tener? (modelo asimilacionista, compensatorio, intercultural...). 3. Conocer los datos que definen la práctica educativa habitual de los y las maestras del centro: experiencia multicultural, formación intercultural, actividades interculturales desarrolladas y forma de trabajo. 4. Averiguar la predisposición del profesorado de la escuela a cambiar su práctica educativa para conseguir lograr un currículum intercultural: disposición a modificar los currículos, adoptar y adaptar metodologías y hábitos de trabajo y relacionarse con los entornos familiares del alumnado inmigrante.

\subsection{VALIDACIÓN DEL CONTENIDO}

Se presentó la versión final a juezas externas, expertas en metodología de investigación y se pasó una prueba piloto en el propio centro, con criterios de comprensión y efectividad para validar el contenido y que, después de diversas modificaciones, quedó como se muestra en el siguiente punto.

\section{2.- DISEÑO CUESTIONARIO}

\section{CUESTIONARIO}

$\mathrm{N}^{\circ}$

1) Nombre (opcional)

\section{2) Edad}

$\square$ Menos de 30 años $\square$ Entre 30 y 40 años

$\square$ Entre 41 y 50 años $\square$ Más de 50 años

\section{3) Sexo}

$\square$ Hombre $\square$ Mujer

4) ¿De qué curso es profesor/as jefe?

\section{Educación Preescolar}

$\square$ Prekinder $\square$ Kinder

\section{Educación Básica}

$\begin{array}{llll}\square 1^{\circ} & \square 2^{\circ} & \square 3^{\circ} & \square 4^{\circ} \\ \square 5^{\circ} & \square 6^{\circ} & \square 7^{\circ} & \square 8^{\circ}\end{array}$

\section{Educación Media}

$\square 1^{\circ} \quad \square 2^{\circ} \quad \square 3^{\circ} \quad \square 4^{\circ}$


5) ¿Qué porcentaje aproximado de alumnado procedente de otros países u otras culturas asiste a su aula?

$\square$ Menos de un $5 \% \quad \square$ entre un 5 y un $10 \% \quad \square$ entre un 10 y un $20 \%$

$\square$ Entre un 20 y un $30 \%$ 口 entre un 30 y un $40 \%$ 口 más de un $40 \%$

6)¿Cuáles son los grupos culturales representados en su aula? Señala grupo étnico-cultural $\mathbf{y ~}^{\mathbf{0}}$ de personas

7) ¿De que tipo son las asignaturas que imparte habitualmente?

$\square$ Matemáticas y tecnología $\quad \square$ Educación física y artística

$\square$ Lenguaje $\quad \square$ Ciencias sociales y Ciencias naturales $\square$ otras

8) ¿Cuál es su experiencia en la enseñanza en aulas multiculturales?

$\square$ Menos de 1 año $\square$ entre 1 y 3 años $\square$ entre 3 y 5 años $\square$ más de 5 años

9) ¿Cómo definiría su experiencia en esta nueva realidad educativa multicultural?

$\square$ Globalmente positiva $\quad \square$ No aprecio la diferencia

$\square$ Globalmente negativa $\quad \square$ No me lo he planteado

$\square$ Otras percepciones

10) ¿Cuál de las siguientes afirmaciones cree que define mejor el papel que le corresponde a la escuela en este nuevo contexto educativo multicultural?

$\square$ Entregar al alumnado extranjero la suficiente competencia lingüística para que se incorpore a nuestro sistema educativo lo antes posible.

$\checkmark$ Compaginar su integración en nuestro sistema educativo con clases en su lengua materna.

$\square$ Habilitar clases especiales donde se trabaje en su propia cultura con alumnado de su mismo grupo étnico.

$\square$ Integrarlo en nuestro sistema educativo incorporando elementos característicos de su bagaje cultural.

11)¿Cuál de los siguientes objetivos cree que deberá apoyar la escuela para este nuevo tipo de alumnado?

$\square$ Intentar olvidar, en todos los ámbitos, cualquier rasgo cultural diferente del nuestro a fin de incorporarse mejor a nuestro sistema educativo.

$\square$ Dejar el cultivo de la cultura propia para el ámbito extraescolar y mirar de adoptar el nuestro en cualquier situación.

$\square$ Intentar integrarse al grupo aportando elementos culturales propios.

$\square$ Procurar rodearse siempre de compañeras y compañeros de su mismo grupo étnico a fin de preservar su identidad cultural. 


\section{2) ¿Con cuál de las siguientes afirmaciones se identifica más?}

$\square$ La escuela debe ayudar a este tipo de alumnado a adaptarse a nuestra cultura aunque ello lleve consigo el renunciar a la de origen.

$\square$ La escuela debe potenciar que el nuevo alumnado conozca nuestra cultura sin renunciar a la suya.

$\square$ Sería mejor habilitar escuelas (o aulas) especiales para alumnado de diferentes grupos étnicos donde se cultive sólo su cultura de origen.

$\square$ No creo que sea necesario plantear ningún cambio.

13) ¿Cree que el alumnado minoritario ha de tener la oportunidad de seguir cultivando su cultura y lengua originaria?

$\square$ No, lo mejor sería habituarse lo antes posible a nuestras lenguas

$\square$ Sí, ya que si no perderían su identidad cultural

$\square$ No, si no, les será muy difícil cambiar sus referentes por otros

$\square$ Sí, ya que la escuela debe incorporar elementos culturales diversos.

14) Si opina que han de "seguir cultivando" su cultura y lengua propias, señale dónde se les debiera atender:

$\square$ Privadamente (familia y/o comunidad);

$\square$ En la escuela;

$\square$ A través de organismos o iniciativas sociales diversas.

$\square$ Otros (especificar)

15) ¿Cree que el cultivo de su cultura y/o lengua originaria beneficiaría la integración de este tipo de alumnado? Señale la razón

$\square$ No $\quad \square$ Sí

$\square$ Porque así les costaría más encontrarse a gusto entre nosotras/os.

$\square$ Porque su incorporación debe producirse sumando su bagaje cultural

$\square$ Porque lo que deben hacer es potenciar el cultivo de la lengua y cultura autóctona

$\square$ Porque así no percibirían el "choque" cultural tan profundamente.

16) ¿Cree que la escuela debería hacer una reflexión sobre el tipo de proyecto educativo curricular que debe ofrecer a raíz de su multiculturalidad? Señale la razón.

$\square$ No $\square$ Sí

$\square$ El centro debe hacer lo mismo para todos/as igual.

$\square$ Porque la situación es muy diferente y no sirven los mismos enfoques.

$\square$ Porque las directrices del Ministerio de Educación no nos lo exigen.

$\square$ Porque se debe aprovechar esta riqueza cultural que llega.

$\square$ Otra (especificar) 
17) ¿Cree que es conveniente que la familia del alumnado inmigrante participe en la vida escolar con aportaciones culturales? Señale la razón.

$\square$ No $\square$ Sí

$\square$ Porque desconociendo nuestro sistema escolar no es conveniente.

$\square$ Porque ante la falta de materiales culturales ellas pueden aportar algunos.

$\square$ Puesto que tampoco participan las familias de los autóctonos.

$\square$ Porque será un primer paso para integrarse en el barrio y la sociedad.

$\square$ Otra (especificar)

18) ¿Cree que la escuela debe compensar el déficit lingüístico y cultural con que acceden a nuestro sistema educativo este tipo de niñas y niños? Señale la razón.

$\square$ No $\square$ Sí

$\square$ Este alumnado lo que debería hacer es un esfuerzo mayor para seguir el ritmo marcado.

$\square$ Porque no parten en las mismas condiciones y sería injusto.

$\square$ Porque no hay ningún déficit que subsanar.

$\square$ Porque tenemos un programa previsto para llevar a cabo esta tarea.

$\square$ Otra (especificar)

19). ¿Cuáles cree que deberían ser las lenguas de aprendizaje de este tipo de alumnado?

$\square$ La lengua vehicular.

$\square$ Su lengua materna.

$\square$ Ambas.

20). ¿Cree que el alumnado minoritario rendiría más si fuese atendido en clases especiales? Señale la razón o razones.

$\square$ No $\quad \square \mathrm{Si}$

$\square$ Porque estaría muy desmotivado.

$\square$ Porque seguiría su propio ritmo y no perdería el hilo.

21) ¿Ha participado en algún programa de formación docente específico para este tipo de alumnado? En caso afirmativo, señale de que tipo.

$\square$ Sí $\square$ No

$\square$ cursos $\square$ jornadas $\square$ seminarios $\square$ formación permanente $\square$ otro tipo 


\section{2) ¿Considera que su formación para trabajar en entornos multiculturales es suficiente?}

$\square$ Supongo que no, pero la verdad es que no me lo he planteado.

$\square$ Sí, porque he ido aprendiendo con la práctica multicultural.

$\square$ Sí, porque he realizado formación específica recientemente.

$\square$ No es necesaria una formación específica, ya que los niños y niñas son iguales en todo el mundo.

23). ¿Cree que el profesorado, en general, posee formación pedagógica específica para enseñar a este tipo de alumnado? Señale la razón.

$\square$ Sí $\square$ No

$\square$ Ahora ya aprenden asignaturas específicas en pedagogía.

$\square$ Todo el profesorado dice que faltan estrategias.

$\square$ Porque se posee suficiente formación pedagógica para ejercer como docente en cualquier situación.

$\square$ Porque hay demasiados problemas derivados de lo mismo.

24) ¿Cree necesario que el profesorado que no tiene alumnado minoritario reciba también formación específica sobre educación intercultural?

$\square$ No, porque si no tiene alumnado foráneo no necesita este tipo de formación.

$\square$ Sí, porque la interculturalidad es una mejora para todo el mundo.

$\square$ No, ya tiene suficiente formación pedagógica para afrontar cualquier situación.

$\square$ Sí, porque además de la escuela, la sociedad es ya multicultural.

25) ¿Realiza algún comentario sobre el país de origen, cultura, lengua etc... cuando llega una persona nueva al aula?

$\square$ No, para no dar relevancia a la "diferencia" de la persona nueva.

$\square$ Sí, como forma de dar la bienvenida al-la recién llegada.

$\square$ No, porque debo seguir un temario y no coincide con lo que toca.

$\checkmark$ Sí, porque es una forma de enriquecernos todas y todos.

\section{6) En las semanas culturales, ¿Realiza alguna actividad multicultural?}

$\square$ No, si no es que toca realizar algo de ese tipo.

$\square$ Sí, porque es un buen momento para trabajar interculturalmente.

$\square$ No, para no reforzar la sensación de confusión cultural.

$\square$ Sí, pinceladas folklóricas que dan mucho juego.

27) ¿Realiza habitualmente algún comentario sobre países, culturas, lenguas diferentes aunque no tengas ningún/a representante de los mismos

$\square$ No, no tiene sentido si todo el alumnado es de la misma cultura.

$\square$ Sí, porque aunque en el aula no sea el caso, en la sociedad sí.

$\square$ No, porque debo seguir un temario y no innovar unilateralmente.

$\square$ Sí, porque es una forma de enriquecernos todas y todos. 
28) ¿Detecta elementos negativos hacia culturas diferentes a la occidental en los libros de texto?

$\square$ Sí, muy a menudo detecto estereotipos y prejuicios culturales.

$\square$ No, pero es que los libros ya están revisados antes de sacarlos.

$\square$ Sí, en alguna ocasión noto algún detalle xenófobo.

$\square$ No, el contenido me parece correcto.

29) ¿Procura añadir elementos positivos y eliminar contenidos discriminatorios de los libros de texto?

$\square$ No, bastante trabajo tengo con seguirlos y acabarlos sin tocar nada.

$\square$ Sí, procuro revisar el contenido y modificarlo si es el caso.

$\square$ No, porque no veo que hoy día haya contenidos discriminatorios.

$\square$ Sí, de hecho, reviso las editoriales para quedarme con la menos "mala".

30) ¿Habitualmente prepara sus clases (programas de asignatura, unidades didácticas...) solo/a o prefiere coordinarse con otras personas?

$\square$ Solo/a siempre, ya que así trabajo mejor.

$\square$ Con compañeras y /o compañeros esporádicamente.

$\square$ Con otras profesoras y profesores siempre que puedo.

$\square$ Ya tengo el material preparado de otras clases.

31) ¿Cree que lo más efectivo es que ajuste la educación de su propio alumnado dependiendo de cada caso concreto?

$\square$ Sí, ya que es la manera de lograr un resultado más positivo.

$\square$ No, ya que prefiero no discriminar a nadie y quiero que todos/as hagan lo mismo.

$\square$ Depende, sólo en casos extremos, ya que representa un trabajo extra muy importante.

$\square$ Otras opciones (especificar)

32) ¿Cuál es su opinión sobre el trabajo en equipo en la práctica educativa?

$\square$ Pienso que se pierde demasiado tiempo trabajando en grupo.

$\square$ Favorable, porque se tienen en cuenta otros puntos de vista.

$\square$ Creo que no acaba de seguirse un camino claro por dar voz a todo el mundo.

33) ¿Estaría dispuesta/o a cambiar su forma de trabajar en el aula? Señale el motivo.

$\square$ No, ya que llevo mucho tiempo trabajando de una forma y no creo que pudiera cambiar.

$\square$ Sí, siempre que no sea un cambio radical.

$\square$ No, porque el alumnado ya se ha habituado a un sistema de trabajo.

$\square$ Sí, no me importa modificar mi práctica docente, pienso que es bueno innovar.

$\square$ Otros: 
34) ¿Aceptaría modificar el P.E.I. para lograr una mejora de sus objetivos respecto a dar entrada a nuevos enfoques culturales en las aulas? Señale el motivo.

$\square$ No, no veo la necesidad de cambiar algo que por consenso se ha creído lo mejor para todo el alumnado.

$\square \mathrm{Si}$, siempre que no fueran cambios drásticos.

$\square$ No, ya que apenas hay tiempo para llevara a cabo lo que ahora hay.

$\square$ Sí, me parece una razón suficiente para rehacer nuestro proyecto.

$\square$ Otros

35) ¿Estaría dispuesto a incorporar material educativo y/o eliminar otro del currículum? Señale el motivo.

$\square$ No, ya que nos ha costado mucho decidir una línea como para cambiar.

$\square$ Sí, siempre que fuera por consenso de todo el profesorado.

$\square$ No, el material educativo hoy día ya está muy "cuidado".

$\square$ Por supuesto, no podemos trabajar el mismo material siempre, sobre todo cuando la situación ha cambiado tanto.

$\square$ Otros

36) ¿Vería factible modificar el horario establecido actualmente para adecuarlo a un nuevo enfoque educativo intercultural? Señale el motivo

$\square$ No, ya ha costado cuadrar el que hay como para cambiar

$\square$ Sí, si va a representar una mejora para el alumnado

$\square$ No, ya está bien de que recaigan todos los trabajos de adecuación sobre el profesorado

$\square$ Sí, me interesa introducir este nuevo enfoque aunque me cueste algún esfuerzo extra

37) ¿Sería capaz de supeditar sus objetivos iniciales a un proyecto común más amplio y culturalmente innovador? Señale el motivo.

$\square$ No, pienso que debemos ser consecuentes con el proyecto inicial de centro.

$\square$ Sí, cualquier modificación que me lleve a mejorar es bienvenida.

$\square$ No, prefiero que de las innovaciones educativo-culturales se hicieran cargo personas específicas.

$\square$ Sí, si me proporcionan material elaborado.

38) ¿Le importaría modificar las unidades que tenía programadas para impartir sus asignaturas, o crear nuevas, para recoger un tipo de enfoque educativo más intercultural?

$\square$ No, es más, pienso que debemos modificar cada curso para avanzar.

$\square$ La verdad es que no veo la necesidad de modificar algo que se prepara para todo el alumnado, sin distinciones.

$\square$ No, siempre que no sean cambios drásticos, sino pequeñas cosas.

$\square$ Sí, cada vez se pide más al profesorado, ya hacemos bastante. 
39) ¿Estaría dispuesta/o a buscar material educativo de otras culturas para dar respuesta a estas nuevas necesidades de su alumnado?

$\square$ No, pienso que no es mi trabajo, hay otras personas para eso.

$\square \mathrm{Si}$, aunque mejor si alguien lo hace (Editoriales, Ministerio Educación...).

$\square$ No, bastante tenemos con intentar llegar a cumplir el temario actual.

$\square$ Sí, me parece una buena idea para inmiscuirnos más en el cambio.

40) ¿Cree que le serviría tener contacto con el entorno familiar del alumnado inmigrante para que le proporcionase materiales diversos sobre su propia cultura?

$\square$ Poco, lo que me aportarían dudo que fuera educativo para nosotros/as.

$\square$ Mucho, ante la falta de materiales culturales diversos en la escuela las familias pueden aportar información válida.

$\square$ No podría ser, puesto que tampoco participan las familias de los autóctonos.

$\square$ Otras razones

41) ¿Cree que sería capaz de discriminar los valores más positivos para su alumnado de material cultural nuevo?

$\square$ No, o al menos no lo tengo claro, así que prefiero no tener que decidir nada.

$\square$ Sí, guiándome por el sentido común y valores universales.

$\square$ No, si fuera tan fácil ya estarían incluidos en los temarios.

$\square$ Sí, porque todas los aportes son igual de válidos, no hay que discriminar.

\subsection{ANÁLISIS DE DATOS}

El análisis se realizó mediante la aplicación del programa informático para datos de tipo cuantitativo SPSS para Windows, al cual se introdujeron las diversas variables, se codificaron y se procedió a crear la matriz de respuestas mediante el vaciado de los cuestionarios. Se realizó un análisis de tipo descriptivo, obteniendo tablas de frecuencias de cada una de las variables para permitir su comentario particular. También se utilizaron tablas de contingencia para cruzar diversas variables entre ellas y permitir una mayor comprensión de la situación. El siguiente Gráfico 1 permite visualizar los resultados finales del análisis. Cada barra representa un cuestionario y un maestro o maestra. El máximo nivel de sensibilidad sería en 120 puntos. Así, nos encontramos con que 5 miembros del profesorado tenían una sensibilidad baja (menos de 60 puntos), 7 personas una sensibilidad media y 9 personas una sensibilidad alta. Con 4 maestros de este último grupo se formó el equipo de trabajo. 


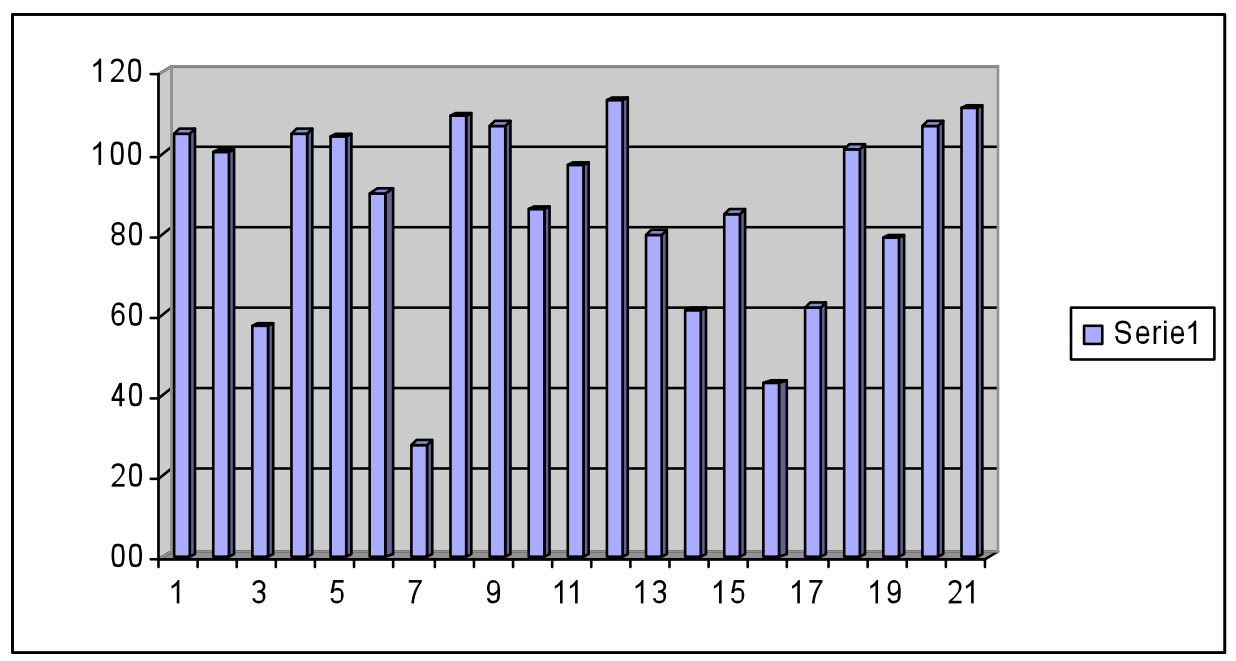

\section{RESULTADOS Y CONCLUSIONES}

Solamente a modo de ejemplo, se presentan los resultados generales, según las dimensiones planteadas a la luz del marco teórico y la conclusión general del trabajo de investigación.

Sobre el rol de la escuela: En este apartado queríamos reflejar, por las respuestas recogidas, cómo era el enfoque educativo que el profesorado pensaba que debía de seguir la escuela y también dejar patente cuál era el modelo que seguía en aquellos momentos. Curiosamente, había un mayoritario apoyo a las cuestiones planteadas como características de la educación intercultural (Jordán, 1996; y Besalú, 2002): reflexionar sobre la práctica educativa, integrar sumando, adicionar cultura y lengua de origen etc. Por otro lado, se contestó mayoritariamente en positivo a las preguntas que plantean principios básicos de la educación compensatoria y todavía de un asimilacionismo más radical e incluso un cierto segregacionismo. Es decir, proteccionismo del recién llegado (en la línea de identificar diferencia con deficiencia), pero a la vez del grupo autóctono, puesto que se defendía el "separar" con la excusa de adecuar el proceso de enseñanza-aprendizaje individualmente y prevalecía el conocimiento de la lengua vehicular por encima de cualquier otra premisa. Este último comentario está muy de acuerdo con el que habíamos reflejado en el marco teórico en lo referente a este tema y que han puesto de relieve varias investigaciones (Bartolomé, 1997a; Colectivo AMANI, 2005).

Sobre el papel del profesorado: En esta dimensión queríamos establecer si también en estos apartados encontraríamos un grupo de docentes que, de nuevo, apoyaran similares conclusiones que otras investigaciones más generales habían sacado a la luz (Jordán, 1996 y Bartolomé, 1997a). Efectivamente, veíamos que, en cuanto a la formación, la mayoría de los maestros pensaban que ésta es muy importante para todo el profesorado, pero la realidad es que sólo dos personas habían llevado a cabo alguna actuación 
específica en este sentido. Contrariamente, declaraban después de que con la práctica ya tenían suficiente capacidad pedagógica como para encararlo con éxito y, aunque es cierto que hemos defendido en el enfoque teórico que la práctica en entornos multiculturales mejora la práctica docente, no parece que esta postura sea suficiente para encarar una transformación curricular con éxito. También es importante resaltar que, en las preguntas sobre la práctica educativa, parecía que había una buena disposición para el trabajo en grupo por encima del trabajo individual, imprescindible como estrategia para desarrollar un modelo de educación intercultural efectivo en los centros.

Sobre la perspectiva de futuro: En esta dimensión establecimos un grupo de sub-dimensiones donde se encontraría la respuesta última que prácticamente más nos interesaba, puesto que se vio, globalmente, qué disposición demuestra la escuela como institución y el profesorado en particular a implicarse en el cambio educativo que les planteábamos. Las primeras contestaciones (preguntas sobre actividades interculturales desarrolladas), parecían demostrarnos que estaríamos en el primer estadio del proceso de cambio curricular de Banks (2004), pero ya apuntando hacia el segundo modelo, en la medida que ya había una tanto por ciento elevado de docentes que confesaban actuaciones de tipo intercultural habitualmente, es decir, que nos encontraríamos entonces en el tipo de currículum aditivo que nos va muy bien para empezar a avanzar hacia el nuevo estadio que queríamos llegar a conseguir y que sería el denominado de transformación. En el segundo grande bloque de preguntas, se demandó al profesorado sobre actuaciones específicas a realizar y su disposición a hacerlas. Evidentemente, estamos hablando aquí de las adecuaciones del segundo y tercer nivel de concreción curricular, imprescindibles para conseguir llegar no sólo al modelo de transformaciones antes referido, sino todavía más allá y conseguir modificar el currículum global del centro añadiendo las relaciones con las familias del alumnado. Ciertamente, y con diferentes porcentajes, se hizo una lectura general que nos indicaba que la disposición existía, pero que, de manera más o menos explícita, el profesorado supeditaba este cambio en su práctica educativa, al hecho de disponer de recursos elaborados e indicaciones precisas.

Como conclusión, y de acuerdo con la constante general en el sistema educativo español, vemos que teóricamente se aceptan y apoyan los enfoques más progresistas e innovadores, pero en la práctica diaria aterrizaban de otra manera en la realidad.

\section{REFERENCIAS BIBLIOGRÁFICAS}

Aranda, V. (2011). Reflexión y análisis de políticas y prácticas innovadoras a la luz de las representaciones sociales y de la necesidad de una educación intercultural en la formación inicial docente. Estud. Pedagógicos, vol. 37, n. 2, 301-314.

Banks, J. A., Mcgee Banks, Ch. (2004). Multicultural Education. Issues and perspectives. USA: Wiley Education.

Bartolomé, M. (1997a). Panorámica general de la investigación sobre educación intercultural en Europa. Revista de Investigación Educativa, vol. 15, n. 1, 7-28.

Bartolomé, M. (Coord). (1997b). Diagnóstico a la escuela multicultural. Barcelona: Cedecs.

Besalú, X. (2004). La formación inicial en interculturalidad. La formación del profesorado en Educación Intercultural (pp. 49-92). Madrid: Los libros de la Catarata.

Buxarrais, M. R. (1997). La formación del profesorado en educación en valores. Propuesta y materiales. Bilbao: Desclée De Brower. 
Carr, W., Kemmis, S. (1988). Teoría crítica de la enseñanza. Barcelona: Martínez Roca.

Centro de investigación y documentación educativa CIDE. (2005). La atención al alumnado inmigrante en el sistema educativo en España. Madrid: MEC.

Colectivo AMANI. (2005). Educación intercultural en el ámbito de la educación formal. Congreso Internacional de Educación intercultural. 13, 14 y 15 de abril de 2005. Córdoba: FT-UGT.

Colectivo IOÉ. (1999). La educación intercultural a prueba. Granada: CIDE, Laboratorio de Estudios Interculturales.

Díaz Aguado, M. (2003). Educación intercultural y aprendizaje cooperativo. Madrid: Ediciones Pirámide.

Ferrao Candau, V. M. (2010). Educación intercultural en América Latina: distintas concepciones y tensiones actuales. Estud. Pedagógicos, vol. 36, n. 2, 333-342.

González Mediel, O. (2008). Cap a un currículum intercultural a Primària. La investigació-acció com a metodologia i la cançó com a desencadenant del canvi. Barcelona: Universitat de Barcelona. Departament de Mètodes d'Investigació i Diagnòstic en Educació.

Jordán, J. (1996). Propuestas de educación intercultural para profesores. Barcelona: CEAC S.A.

Jordán, J. (2004). La formación permanente del profesorado en Educación Intercultural. En Jordán, J. A.; Besalú, X., Bartolomé, M., Aguado, M. J., Moreno, C., y Sanz, M. (Coord.), La formación del profesorado en Educación Intercultural (pp. 11-48). Madrid: Los libros de la Catarata.

Rubio, M. (2009). El desarrollo de la competencia comunicativa intercultural en la formacion inicial docente. Estud. Pedagógicos, vol. 35, n. 1, 273-286.

Sabariego, M. (2003). La formación del profesorado en educación intercultural. Temps d'Educació, n. 27. 